\title{
Omalizumabe no tratamento de urticária solar refratária aos anti-histamínicos: relato de caso e revisão da literatura
}

\author{
Omalizumab in the treatment of solar urticaria refractory to antihistamines: \\ a case report and literature review
}

Bárbara Martins de Aquino ${ }^{1}$, Alex Eustáquio de Lacerda ${ }^{1}$,
Inês Cristina Camelo Nunes ${ }^{1}$, Luis Felipe Chiaverini Ensina ${ }^{1}$

\section{RESUMO}

A urticária solar é uma forma rara de urticária crônica induzida (UCInd). Os sintomas se iniciam minutos após a exposição ao sol e persistem por até 2 horas, interferindo nas atividades diárias do paciente, e consequentemente na sua qualidade de vida. $\mathrm{O}$ omalizumabe, anticorpo monoclonal anti-IgE já aprovado para o tratamento da urticária crônica espontânea, tem sido utilizado no tratamento das urticárias crônicas induzidas com boa resposta, inclusive na urticária solar. Neste artigo, relatamos um caso de urticária solar refratária aos anti-histaminicos, sua evolução após o uso do omalizumabe, e fazemos uma breve revisão da literatura sobre o tema.

Descritores: Urticária crônica, omalizumabe, dermatopatias.

\section{Introdução}

Urticária solar é uma fotodermatose rara, caracterizada pelo surgimento de urticas associadas a prurido ou sensação de queimação na pele, minutos após exposição à radiação UVA, UVB ou luz visível, desaparecendo cerca de 1 a 2 horas após fim da exposição ${ }^{1}$.

Considerada uma forma de urticária crônica induzida, aquelas desencadeadas por estímulos específicos, essa fotodermatose tem mecanismo fisiopatológico provavelmente IgE mediado, desencadeado por um fotoalérgeno ativado por particular espectro de luz ${ }^{2}$. Situações de exposição solar intensa e prolongada

\section{ABSTRACT}

Solar urticaria is a rare form of chronic inducible urticaria. Symptoms start minutes after exposure to the sun and persist for up to 2 hours, interfering with the patients' daily activities and, consequently, their quality of life. Omalizumab, a monoclonal anti-lgE antibody already approved for the treatment of chronic spontaneous urticaria, has been used in the treatment of chronic inducible urticaria, including solar urticaria, with good response. In this article, we report a case of solar urticaria refractory to antihistamines, show the patient's evolution after the use of omalizumab, and briefly review the literature on the subject.

Keywords: Chronic urticaria, omalizumab, skin diseases.

podem resultar em quadros graves, com desenvolvimento de sintomas sistêmicos e anafilaxia ${ }^{3}$.

Embora uma parcela de indivíduos com urticária solar responda às terapias de primeira linha (antihistamínicos de segunda geração associados ou não a fotoprotetores), há pacientes com doença grave refratária aos anti-H1 que requerem outros tratamentos, incluindo fototerapia, imunossupressores e imunobiológicos ${ }^{4}$.

O omalizumabe, anticorpo anti-lgE recombinante humanizado, já aprovado para o tratamento da urticária crônica espontânea, é recomendado e tem

1. Universidade Federal de São Paulo, Disciplina de Alergia Imunologia Clínica e Reumatologia, Departamento de Pediatria - São Paulo, SP, Brasil. 
sido utilizado também como terceira linha nas urticárias crônicas induzidas, com resposta favorável ${ }^{5}$. Entretanto, dados sobre sua eficácia para tratamento da urticária solar são escassos, e limitados a pequenas séries e relatos de caso ${ }^{4}$.

\section{Relato do caso}

Paciente do sexo feminino, 20 anos de idade, estudante de enfermagem, apresentava urticas, prurido e sensação de queimação nas áreas da pele descobertas e expostas à luz solar desde os 12 anos. Os sintomas iniciavam cerca de 15-20 minutos após se expor ao sol, com regressão total em até 30 minutos ao final da exposição. Nas crises mais graves, que ocorriam durante intensa exposição solar, além das urticas, a paciente apresentava hipotensão, dispneia e incontinência urinária.

O diagnóstico foi confirmado pelo teste de provocação, com exposição direta à luz solar por 20 minutos, tempo máximo para surgimento dos sintomas referido pela própria paciente. Quinze minutos após o início do teste, ela apresentou urticas e prurido nas áreas da pele exposta (Figuras 1, 2 e 3), com remissão total dos sintomas em 30 minutos após a exposição.

Apesar do tratamento com dose quadruplicada de anti-histamínico de segunda geração, a paciente mantinha os sintomas. Com isso, sua qualidade de vida era extremamente prejudicada, já que não conseguia exercer suas atividades ao ar livre no período diurno devido ao incômodo e constrangimento causados pelas lesões de pele e prurido decorrentes da urticária.

Foi então proposto o tratamento adicional com omalizumabe $300 \mathrm{mg}$ por via subcutânea a cada 4 semanas, em dezembro de 2018. Dentro das duas primeiras semanas após a primeira aplicação, a paciente evoluiu com melhora dos sintomas, permitindoIhe retomar suas atividades habituais ao ar livre no período diurno.

Neste caso, os efeitos da terapia anti-IgE foram avaliados sob o ponto de vista clínico, pois na ocasião das consultas iniciais, o Urticaria Control Test (UCT) na versão para o português do Brasil ainda não havia sido validado. O Chronic Urticaria Quality of Life Questionnaire (CU-QoL), por sua vez, não é realizado rotineiramente em nosso serviço, apenas em pacientes participantes de alguns estudos específicos.

Devido ao bom controle dos sintomas depois da introdução do omalizumabe, a paciente suspendeu

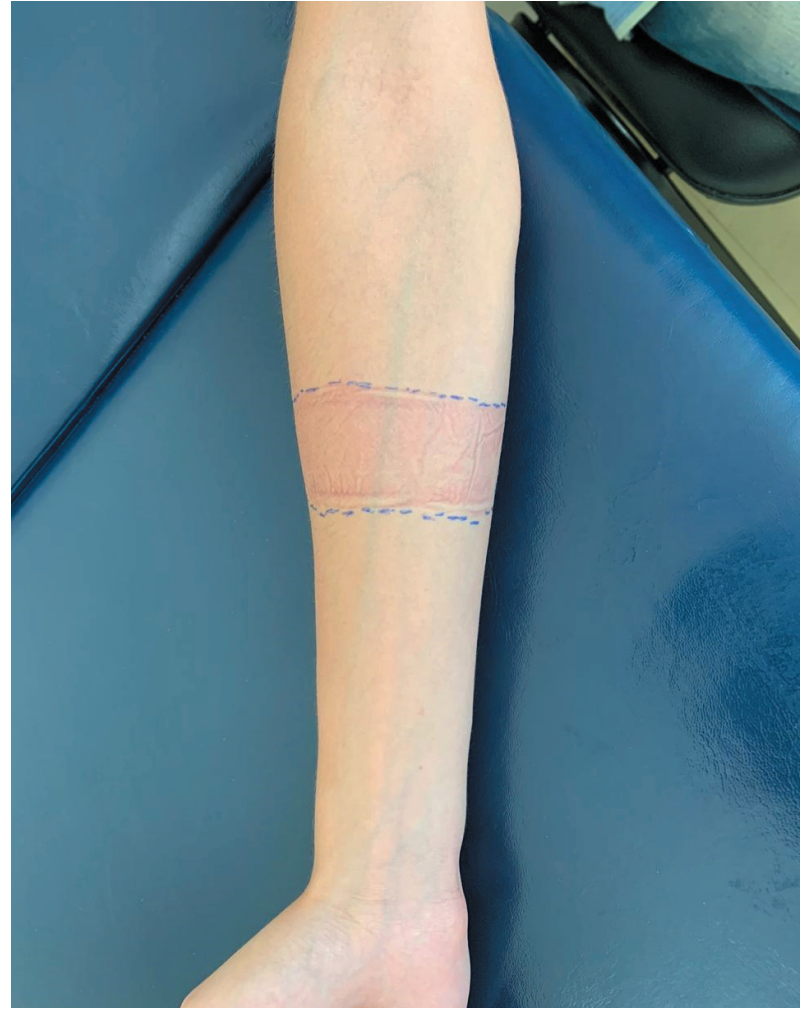

Figura 1

Teste de provocação com exposição à luz solar

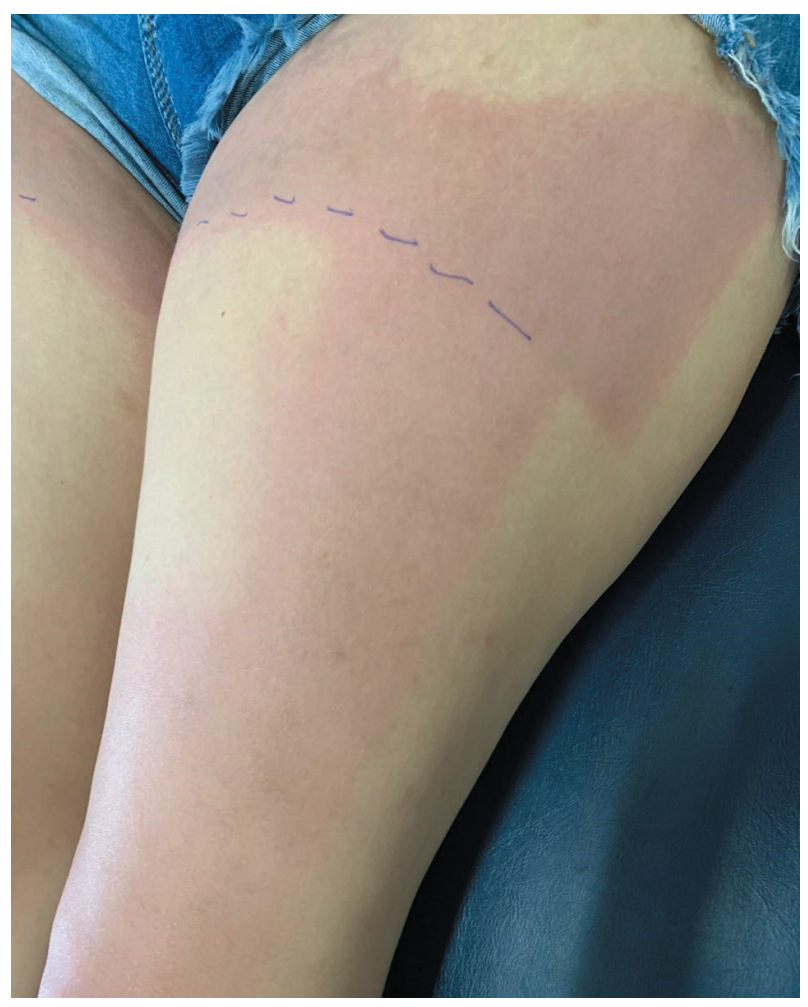

Figura 2

Teste de provocação com exposição à luz solar 


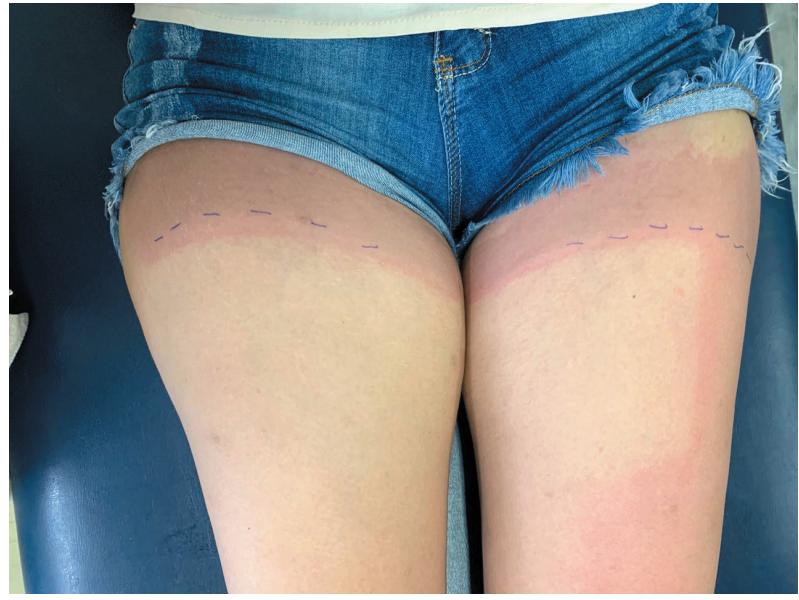

Figura 3

Teste de provocação com exposição à luz solar

por conta própria o anti-histamínico logo após a segunda dose da terapia anti-IgE, mantendo-se controlada da urticária apenas com o omalizumabe. Porém, seis meses depois, voltou a apresentar eritema e prurido nas áreas expostas à luz solar. Por esta razão, foi orientada a reintrodução do anti-histamínico em dose quadruplicada. Após essa medida, a paciente evoluiu novamente com controle dos sintomas.

\section{Discussão}

A urticária é uma condição em que há o surgimento de urticas (lesões com edema central de tamanho variável cercado ou não por eritema reflexo, coceira ou sensação de queimação e natureza fugaz, com a pele retornando ao normal em até 24 horas) associadas ou não ao angioedema (edema repentino, pronunciado e deformante, da derme e subcutâneo ou membranas mucosas, acompanhado de dor, queimação ou prurido, e com resolução em até 72 horas). Sua classificação é baseada na duração dos sintomas (crônica quando é superior a 6 semanas) e na presença ou ausência de fatores desencadeantes específicos (induzidas ou espontâneas, respectivamente $)^{5}$.

As urticárias crônicas induzidas (UCInd) são aquelas em que urticas e ou angioedema surgem após um estímulo específico, que pode ser físico (urticária ao calor ou frio, urticária de pressão tardia, urticária vibratória, dermografismo e urticária solar) ou não físico (urticária colinérgica, urticária de contato e aquagênica) $)^{5,6}$. A prevalência estimada das UCInd é de $0,5 \%$ na população geral 6 . A urticária solar é um tipo raro de UCInd, que representa $7 \%$ das fotodermatoses $^{7}$, e até cerca de $0,5 \%$ de todas as urticárias ${ }^{1}$. Afeta predominantemente o sexo feminino, principalmente nas terceira e quarta décadas de vida, podendo estar associada a outras urticárias crônicas em até $16 \%$ dos casos, e à atopia em cerca de $30 \% 1,8,9$.

O quadro característico da urticária solar é o surgimento de urticas em áreas não cobertas do corpo, geralmente em até 15 minutos após a exposição à luz solar, e que têm remissão espontânea em no máximo 24 horas. Entretanto, existem casos descritos de lesões que aparecem horas após a exposição à luz, levando tempo superior a 24 horas para remissão, em regiões não expostas do corpo (tecidos finos), e até mesmo se manifestando somente com angioede$\mathrm{ma}^{10-12}$. No presente caso, a paciente apresentava um rápido surgimento das lesões, com rápida resolução do quadro, mesmo sem a utilização de medicações.

A fisiopatologia da urticária solar não é totalmente conhecida, porém a hipótese atual é de que se trate de um mecanismo que envolve uma reação de hipersensibilidade IgE-mediada ou do tipo 1 de Gell e Coombs. Após a exposição solar, uma molécula precursora conhecida como cromóforo seria ativada por um espectro de luz específico, tornando-se um fotoalérgeno imunologicamente ativo. Estes autoalérgenos se ligariam à IgE específicas nos mastócitos, provocando a desgranulação e liberação de mediadores inflamatórios, principalmente a histamina, responsáveis pelos sintomas.

Atualmente, a urticária solar é classificada em dois tipos, segundo o mecanismo envolvido: no tipo I, há a presença de anticorpos IgE específicos contra cromóforos anormais presentes somente em pessoas acometidas; no tipo II, há a presença de IgE específica contra cromóforos normais. Esta diferença é evidenciada no teste de transferência sérica passiva de pacientes com urticaria solar para controles saudáveis, em que somente controles que receberam a transferência de pacientes com o tipo II têm o teste positivo, com o surgimento de urticária ${ }^{1,8,10,13}$.

Os indivíduos com urticária solar reagem a diferentes espectros de luz (UVA, UVB, Luz Visível) e a gravidade do quadro depende do tempo, intensidade e área exposta ao espectro de luz específico. Regiões da pele que são frequentemente expostas à luz tendem a ser menos sensíveis para o desenvolvimento das lesões, num fenômeno de tolerância mais conhecido como "hardening", e alguns comprimentos de onda de radiação (geralmente os mais longos) 
podem inibir a reação causada por outros (geralmente os mais curtos), num mecanismo conhecido como duplo espectro de ação ${ }^{1,6,10}$. A maioria dos pacientes tem como gatilho os espectros de luz visível, UVA e a associação de luz visível e UVA ${ }^{10}$. Quadros com sintomas sistêmicos e anafilaxia podem ocorrem com a realização de "banhos de sol" ou exposição de grandes superfícies corporais à luz 1,7,8,10. Além da urticária, a paciente apresentou, quando intensamente exposta ao sol (ex. praia), sintomas circulatórios (hipotensão), respiratórios (dispneia) e neurológicos (liberação de esfíncter urinário), caracterizando anafilaxia e necessidade de tratamento urgente ${ }^{15}$.

Outras fotodermatoses (erupção polimorfa à luz, erupção fotoalérgica, fototoxicidade por droga, Lupus eritematoso cutâneo) e urticárias induzidas (por calor e colinérgica) podem ter sinais e sintomas que se confundem com a urticária solar, e por isso é fundamental a confirmação diagnóstica com o teste de provocação6,7. O fototeste ou fotoprovocação é realizado com a exposição da superfície cutânea (20 a $25 \mathrm{~cm}^{2}$ ) a uma fonte emissora de diferentes espectros de luz (UVB, UVA, luz visível) com diferentes doses (UVA: 2,4-3,3-4,2-5,1-6 J/cm² e UVB 24-3342-51-60 mJ/ $\mathrm{cm}^{2}$ ). O teste é considerado positivo caso haja surgimento de urticas após 10 minutos da exposição6,10. Entretanto há pacientes que não apresentam positividade no fototeste ou mesmo só reagem a espectros de luz combinados (ex. UVA + luz visível) ${ }^{9}$. A determinação do espectro de luz e da dose necessária para desencadear as lesões é importante para o tratamento (fototerapia), profilaxia (protetor solar com filtro específico, janelas com bloqueio UV específico) e acompanhamento do tratamento (doses mais altas de exposição sem lesões) ${ }^{8,14}$. A dose eritematosa mínima (DEM) de urticária, que é a menor quantidade de energia efetiva para produzir o surgimento da reação, pode ser determinada expondo, a uma distância de $10 \mathrm{~cm}$ da fonte de luz, uma área de $1 \mathrm{~cm}^{2}$ de pele ${ }^{16}$. Na indisponibilidade de uma fonte de emissão de diferentes espectros de luz, outras opções para fotoprovocação são com lâmpada de projetor de slides (luz visível), luz negra fluorescente (UVB e UVA), luz solar fluorescente (UVB e UVA) e lâmpada infravermelha ${ }^{17}$. Optamos pela confirmação do diagnóstico expondo a paciente à luz natural, uma forma simples e econômica de reproduzir os sintomas, mas que não fornece informações específicas sobre espectro e dose necessária de exposição.

Além do uso de protetores solares, o tratamento inicial da urticária solar segue o mesmo algoritmo re- comendado para as demais urticárias, com anti-histamínicos de segunda geração em doses padronizadas ou até quadriplicadas ${ }^{5}$. No entanto, muitos pacientes não têm o controle adequado dos sintomas, e outros tratamentos foram experimentados, como fototerapia, PUVA, ciclosporina A, bloqueador de receptor de leucotrieno, imunoglobulina, antimaláricos e plasmaferese, com resultados variáveis ${ }^{1,5,8,18}$. Seguindo as recomendações das diretrizes atuais, e considerando possível eficácia e conhecida segurança, optamos por introduzir o omalizumabe ${ }^{5}$.

O omalizumabe é um anticorpo monoclonal humanizado, com a capacidade de se ligar ao IgE circulante e inibir a interação entre o $\operatorname{lgE}$ e o receptor de alta afinidade do mastócito (FcERI), consequentemente diminuindo o IgE livre e reduzindo a expressão do FceRI ${ }^{19}$. Como o mecanismo proposto para o surgimento das lesões na urticária solar é pela ligação do IgE no FceRI do mastócito, a ação do omalizumabe nesta via pode interferir e suprimir o processo de surgimento dos sintomas 20 .

Em um total de 24 publicações que somam 66 pacientes ${ }^{2,3,21-42}$ tratados com omalizumabe, $77,3 \%$ (51) dos indivíduos evoluíram com controle completo, $13,7 \%$ (9) tiveram resposta parcial e 9\% (6) não apresentaram resposta com a medicação. Três pacientes apresentaram controle apenas com a utilização de doses mais altas (> $450 \mathrm{mg} / \mathrm{mês})^{32,33,40}$, e três pacientes foram refratários mesmo com doses de $600 \mathrm{mg} / \mathrm{mês}$, sugerindo que a resposta é individual para cada paciente, e pode ser dose-dependente em casos específicos.

$\mathrm{Na}$ maior série de casos, com 20 pacientes, Morgado-Carrasco e cols. definiram a resposta no controle clínico (RCC) da urticária solar com a pontuação do Escore de Atividade da Urticária (Urticária Activity Score) de sete dias (UAS7) igual a zero. Também avaliaram o Teste de controle da urticária (Urticária Control Test) (UCT controlado > 12) e a presença de efeitos adversos. O RCC foi atingido com doses de $300 \mathrm{mg} / \mathrm{mês}$ em 16 pacientes e com o aumento para $450 \mathrm{mg} / \mathrm{mês}$ em dois, porém mesmo com a progressão da dose para $600 \mathrm{mg} / \mathrm{mês}$, dois pacientes não tiveram resposta. Um terço atingiu o RCC somente após 4 meses de tratamento, demonstrando que além do aumento de dose, pode ser necessário a terapia mais prolongada para atingir o controle da urticária solar.

No presente artigo relatamos o caso de uma paciente com urticária solar refratária ao uso de anti-histamínicos em dose quadriplicada, com re- 
ação grave após a intensa exposição solar e que apresentou controle rápido e duradouro com o uso de omalizumabe. No nosso conhecimento, este é o primeiro caso relatado no Brasil de tratamento eficaz de urticária solar com omalizumabe.

\section{Referências}

1. Botto NC, Warshaw EM. Solar urticaria. J Am Acad Dermatol. 2008;59(6):909-22. doi:10.1016/j.jaad.2008.08.020.

2. Wright $E$, Kurland $E$, Lim HW. Solar urticaria caused by visible light in a 33-year-old male refractory to treatment with omalizumab. Photodermatol Photoimmunol Photomed. 2020;36(4):316-7. doi:10.1111/phpp.12545.

3. de Dios-VelázquezÁ, González-de Arriba M, Beteta-GorritiV, Macías E, Campanón-Toro V, Dávila I.Effectiveness of omalizumab in severe solar urticaria. Ann Allergy Asthma Immunol. 2016;116(3):260-2. doi:10.1016/j.anai.2015.12.023.

4. Snast I, Kremer N, Lapidoth M, Enk CD, Tal Y, Rosman Y, et al. Omalizumab for the Treatment of Solar Urticaria: Case Series and Systematic Review of the Literature. J Allergy Clin Immunol Pract. 2018 Jul-Aug;6(4):1198-1204.e3. doi: 10.1016/j.jaip.2018.02.032.

5. Zuberbier T, Aberer W, Asero R, Abdul Latiff AH, Baker D, BallmerWeber $\mathrm{B}$, et al. The EAACI/GA2LEN/EDF/WAO guideline for the definition, classification, diagnosis and management of urticaria. Allergy. 2018;73(7):1393-414. doi: 10.1111/all.13397.

6. Maurer M, Fluhr JW, Khan DA. How to approach chronic inducible urticaria. J Allergy Clin Immunol Pract. 2018;6(4):1119-30. doi:10.1016/j.jaip.2018.03.007.

7. Chong WS, Khoo SW. Solar urticaria in Singapore: An uncommon photodermatosis seen in a tertiary dermatology center over a 10-year period. Photodermatol Photoimmunol Photomed. 2004;20:101-4.

8. Harris BW, Badri T, Schlessinger J. Solar urticaria. StatPearls [Internet].Disponível em:https://www.statpearls.com/ArticleLibrary/ viewarticle/30885.

9. Pérez-Ferriols A, Barnadas M, Gardeazábal J, de Argila D, Carrascosa JM, Aguilera P, et al. Solar urticaria: Epidemiology and clinical phenotypes in a Spanish series of 224 patients. Actas Dermosifiliogr. 2017;108(2):132-9.

10. Raigosa M, Toro Y, Sánchez J. Urticaria solar. Reporte de un caso y revisión de la literatura. Rev Alerg Mex. 2017;64(3):371-5. doi:10.29262/ram.v64i3.202.

11. Beattie PE, Dawe RS, Ibbotson SH, Ferguson J. Characteristics and prognosis of idiopathic solar urticaria: a cohort of 87 cases. Arch Dermatol. 2003;139(9):1149-54. doi:10.1001/ archderm.139.9.1149.

12. Luther CA, Lim HW. Solar angioedema: A report of a patient and a review of literature. Photodermatol Photoimmunol Photomed. 2019;35(3):187-9. doi:10.1111/phpp.12445.

13. Kojima M, Horiko T, Nakamura Y, Aoki T. Solar urticaria. The relationship of photoallergen and action spectrum. Arch Dermatol. 1986;122(5):550-5. doi:10.1001/archderm.122.5.550.

14. Aguilera P. Inducing light tolerance with narrowband UV-B therapy in solar urticaria. Inducción de fototolerancia con ultravioleta B de banda estrecha en urticaria solar. Actas Dermosifiliogr. 2018;109(10):853. doi:10.1016/j.ad.2018.06.009.

15. Cardona V, Ansotegui IJ, Ebisawa M, El-GamalY, Fernandez Rivas $\mathrm{M}$, Fineman S, et al. World allergy organization anaphylaxis guidance 2020. World Allergy Organ J [Internet]. 2020;13(10):100472. doi:10.1016/j.waojou.2020.10047.

16. Magerl M, Altrichter S, Borzova E, Giménez-Arnau A, Grattan CE, Lawlor $F$, et al. The definition, diagnostic testing, and management of chronic inducible urticarias - TheEAACI/GA(2) LEN/EDF/UNEV consensus recommendations 2016 update and revision. Allergy. 2016 Jun;71(6):780-2.
17. Junior SDD, Azizi GG, Sousa ACM, Lupi O, França AT, Valle SOR. Urticárias crônicas induzidas: atualização do tema. Arq Asma Alerg Imunol. 2020;4(3):305-16.

18. Levi A, Enk CD. Treatment of solar urticaria using antihistamine and leukotriene receptor antagonist combinations tailored to disease severity. Photodermatol Photoimmunol Photomed. 2015;31(6):302-6. doi:10.1111/phpp.12186.

19. Okayama $Y$, Matsumoto $H$, Odajima $H$, Takahagi $S$, Hide $M$, Okubo $\mathrm{K}$. Roles of omalizumab in various allergic diseases. Allergol Int. 2020;69(2):167-7. doi:10.1016/j.alit.2020.01.004.

20. Maurer M, Metz M, Brehler R, Hillen U, Jakob T, Mahler V, et al. Omalizumab treatment in patients with chronic inducible urticaria: A systematic review of published evidence. J Allergy Clin Immunol. 2018 Feb;141(2):638-49.

21. Güzelbey O, Ardelean E, Magerl M, Zuberbier T, Maurer M, Metz $M$. Successful treatment of solar urticaria with anti-immunoglobulin E therapy. Allergy. 2008;63(11):1563-5. doi:10.1111/j.13989995.2008.01879.x.

22. Waibel KH, Reese DA, Hamilton RG, Devillez RL. Partial improvement of solar urticaria after omalizumab. J Allergy Clin Immunol. 2010;125(2):490-91. doi:10.1016/j.jaci.2009.11.007.

23. Metz M, Altrichter S, Ardelean E, Kessler B, Krause K, Magerl M, et al. Anti-immunoglobulin $E$ treatment of patients with recalcitrant physical urticaria. Int Arch Allergy Immunol. 2011;154(2):177-80. doi: 10.1159/000320233.

24. Duchini G, Bäumler W, Bircher AJ, Scherer K. Failure of omalizumab (Xolair $\AA$ ) in the treatment of a case of solar urticaria caused by ultraviolet $A$ and visible light. Photodermatol Photoimmunol Photomed. 2011;27(6):336-7. doi:10.1111/j.16000781.2011.00624.x.

25. Metz M, Ohanyan T, Church MK, Maurer M. Omalizumab is an effective and rapidly acting therapy in difficult-to-treat chronic urticaria: a retrospective clinical analysis. J Dermatol Sci. 2014;73(1):57-62. doi:10.1016/j.jdermsci.2013.08.011.

26. Levi A, TalY, Dranitzki Z, Shalit M, Enk CD. Successful omalizumab treatment of severe solar urticaria in a 6-year-old child. Pediatr Allergy Immunol. 2015;26(6):588-90. doi:10.1111/pai.12441.

27. Arasi S, Crisafulli G, Caminiti L, Guarneri F, Aversa T, Porcaro F, et al. Treatment with omalizumab in a 16-year-old Caucasian girl with refractory solar urticaria. Pediatr Allergy Immunol.2015;26(6):583-5. doi: 10.1111/pai.12413.

28. Müller S, Schempp CM, Jakob T. Failure of omalizumab in the treatment of solar urticaria. J Eur Acad Dermatol Venereol. 2016;30(3):524-5. doi:10.1111/jdv.12922.

29. Terrani I, Bircher AJ, Scherer Hofmeier K. Solar urticaria induced by visible light: successful treatment with omalizumab. Clin Exp Dermatol. 2016;41(8):890-2. doi:10.1111/ced.12951.

30. Moncourier M, Assikar S, Matei I, Souyri N, Couture M, Rigot E, et al. Visible light-induced solar urticaria is improved by omalizumab. Photodermatol Photoimmunol Photomed.2016 Sep;32(5-6):314-16. doi: 10.1111/phpp.12271.

31. Ghazanfar MN, Sand C, Thomsen SF. Effectiveness and safety of omalizumab in chronic spontaneous or inducible urticaria: evaluation of 154 patients. Br J Dermatol. 2016;175(2):404-6. doi:10.1111/ bjd. 14540 .

32. Brüning JH, Ziemer M, Pemler S, Simon JC, Treudler R. Successful treatment of solar urticaria with omalizumab. J Dtsch Dermatol Ges. 2016;14(9):936-7. doi:10.1111/ddg.13017.

33. Baliu-Piqué C, Aguilera Peiró P. Three cases of solar urticaria successfully treated with omalizumab.J Eur Acad Dermatol Venereol. 2016;30(4):704-6. doi:10.1111/jdv.13001.

34. KowalzickL, ThielW, C Bielfeld, Ziegler H, Eickenscheidt L. Partielles Ansprechen einer Lichturtikaria auf Omalizumab [Partial response of solar urticaria to omalizumab therapy]. Hautarzt. 2017;68(6):492-6. doi:10.1007/s00105-016-3913-0.

35. Aubin F, Avenel-Audran M, Jeanmougin M, Adamski H, Peyron JL, Marguery MC, etal.Omalizumab in patients with severe and refractory solar urticaria: A phase II multicentric study. J Am Acad Dermatol. 2016 Mar;74(3):574-5. doi: 10.1016/j.jaad.2015.11.021. 
36. Combalia A, Fernández-Sartorio C, Aguilera P. Refractory solar urticaria successfully treated with omalizumab with normalization of phototest.Actas Dermosifiliogr.2017;108(6):593-4.doi:10.1016/j. ad.2016.11.015.

37. Martinis M, Sirufo MM, Ginaldi L. Solar urticaria, a disease with many dark sides: is omalizumab the right therapeutic response? Reflections from a clinical case report. Open Med (Wars). 2019;14:403-406. doi:10.1515/med-2019-0042.

38. Kieselova K, Santiago F, Henrique M. Incapacitating solar urticaria: successful treatment with omalizumab. An Bras Dermatol. 2019;94(3):331-3. doi:10.1590/abd1806-4841.20198109.

39. Schaffenburg WC, Guerrero KT, Marks SN. Solar urticaria treated with omalizumab. Cutis. 2019;104(1):E4-E5.

40. Snast I, Lapidoth M, Uvaidov V, Enk CD, Mazor S, Hodak E, et al. Real-life experience in the treatment of solar urticaria: retrospective cohort study. Clin Exp Dermatol. 2019 Jul;44(5):e164-e170. doi: 10.1111/ced.13960.
41. Morgado-Carrasco D, Giácaman-Von der Weth M, Fustá-Novell X, Podlipnik S, Pérez-Ferriols A, Aguilera P. Clinical response and long-term follow-up of 20 patients with refractory solar urticaria under treatment with omalizumab. J Am Acad Dermatol. 2019:S01909622(19)30874-6. doi: 10.1016/j.jaad.2019.05.070.

42. Vollono L, Bianchi L, Piccolo A, Mazzilli S, Campione E, Diluvio L. Good things come to those who wait: Successful response of solar urticaria to omalizumab after 1 year of treatment. Photodermatol PhotoimmunolPhotomed.2020;10.1111/phpp.12577.doi:10.1111/ phpp.12577.

Não foram declarados conflitos de interesse associados à publicação deste artigo.

Correspondência:

Bárbara Martins de Aquino

E-mail: barbaraalergia@gmail.com 